Vol. 39 (1989) [81-106]

\title{
NORMALISING ELEMENTS AND RADICALS, I.
}

\author{
E.A. Whelan
}

In this paper we study rings and bimodules with no known one-sided chain conditions, but whose ( $t$ wo-sided) ideals and subbimodules are 'nicely' generated. We define bi-noetherian polycentral $(B P C)$ and bi-noetherian polynormal $(B P N)$ rings and bimodules, large classes of (almost always) non-noetherian objects, and put on record the basic facts about them. Any BPC ring is a BPN ring. In the case of rings we reduce their properties to properties of the prime ideals, and study the d.c.c. on (two-sided) ideals. We define both the artinian and bi-artinian radicals of a BPN ring, and use them to show that for BPN rings the intersections of the powers of both the Brown-McCoy and the Jacobson radicals are zero.

\section{INTRODUCTION}

In this paper we continue the study begun in $[22,24,25]$ of associative rings $R$ with unity (hereafter just 'rings') and of bimodules $B={ }_{S} B_{R}$ over rings $S, R$, in which ideals and subbimodules are 'nicely' generated. For bimodules the condition in which we are mainly interested is: every subbimodule of $B$ is generated by a finite sequence $u_{1}, \ldots, u_{n}$ of elements of $B$ such that, for all $j$ with $1 \leqslant j \leqslant n$,

$$
\sum_{i=1}^{j} S u_{i}=\sum_{i=1}^{j} u_{i} R .
$$

Such a bimodule we call a bi-noetherian polynormal ( $B P N)$ bimodule, for reasons explained in Section 2. A ring $R$ such that ${ }_{R} R_{R}$ is a BPN bimodule is called a $b i$ noetherian polynormal (BPN) ring, and here and elsewhere $[25,24]$ we develop an extensive theory of BPN rings. It is worth noting that, except briefly in Section 5 , we make no assumptions whatever about one-sided ideals.

The paper falls naturally into three parts. In the first (Sections 1 to 3), we cover preliminiaries, and give examples of the rings in which we are interested. This includes listing several new classes of (generally non-noetherian) BPN rings, and discussing results from [27] about the 'stability' of the property under standard ring extensions. We also introduce the class of bi-noetherian polycentral $(B P C)$ rings, which is a strict subclass of the class of BPN rings possessing interesting properties of its own.

Received 6 April, 1987

The results in this paper are adapted from two chapters of the author's PhD thesis [24], and I would like to thank my supervisor Dr. A.R. Camina for his advice, assistance and encouragement. Grateful thanks are also due to Dr A.W. Chatters for his encouragement, comments and other valuable help.

Copyright Clearance Centre, Inc. Serial-fee code: 0004-9729/89 \$A2.00+0.00. 
In the second part (Sections 4 to 6 ) we show that many basic results about commutative noetherian rings and their finitely generated modules extend to this noncommutative setting. In Section 4 we deal-by analogy with the right noetherian case-with a suitable category of bimodules over BPN rings, and a similar one for BPC rings, and show that both categories have various desirable properties, notably that they are abelian. Most of the section involves putting on the record not very deep results (already used elsewhere, for example in [25]), which are very similar to corresponding ones for right noetherian rings, but without which the whole theory of BPN rings cannot be developed. The end of the section, however, initiates a comparison (continued in Section 10) between BPN and BPC rings and left-right noetherian rings, by showing that (under mild faithfulness assumptions) the BPC/and BPN properties of bimodules arise only when the rings in question are themselves BPC or BPN. Then in Section 5 we use the results of Section 4 to show that a ring is BPN if and only if every prime ideal is suitably generated, generalising a well-known result for commutative rings due to I.S. Cohen, and we establish a non-commutative extension of Kaplansky's well-known characterisation [13] of commutative principal ideal rings. In Section 6 we show that a BPN ring has d.c.c. on two-sided ideals if and only if every prime ideal is maximal, almost (but not quite) parallelling the case of commutative rings.

In the third part (Sections 7 to 10) we examine radicals. Section 7 compares BPN rings with (left-right) noetherian ones, to show that BPN rings have both an artinian radical (that is, there is a unique largest left artinian left ideal which is a two-sided ideal coinciding with the unique largest right artinian right ideal), and-unlike the noetherian case-a useful bi-artinian radical (defined as the unique largest ideal with a bi-module composition series). Both radicals have properties closely resembling those of the artinian radical in a noetherian ring.

Then in Section 8 we digress slightly from BPN rings to discuss the Brown-McCoy radical of an arbitary 'associative ring not necessarily with unity', and obtain for it an annihilator characterisation resembling that of the Jacobson radical. This sheds extra light on the fact (noted in Section 4) that the Brown-McCoy radical plays a rôle in the theory of BPN rings and bimodules very like that of the Jacobson radical for right noetherian rings and modules. Then in Section 9 we show that in important ways the Brown-McCoy radical is the 'better-behaved' of the two: in any BPN ring the Brown-McCoy radical $B$ and (hence) the Jacobson radical $J$ satisfy

$$
\bigcap_{n=1}^{\infty} B^{n}=\bigcap_{n=1}^{\infty} J^{n}=0 .
$$

This is the first identification of a large class of non-commutative non-(left-right) noetherian rings which satisfy the Jacobson conjecture. It depends on linking the Brown- 
McCoy and the bi-artinian radicals (Corollary 7.8). Section 10 seeks to put the similarities between the classes of left-right artinian and of left-right noetherian rings and the classes of BPC and BPN rings into a more general context.

In companion papers $[27,28]$ (in preparation) we examine respectively the behaviour of BPN and BPC properties under ring extensions, and further aspects of the theory of BPN and BPC rings, such as completions, graded rings, and the AR property. Some results from [27] are mentioned in Section 3.

\section{TERminology and NOTATION}

Terminology is generally standard, but there are several points worth mentioning. First, the term ring always means an associative ring with unity, and all (bi)modules over rings are assumed to be unital. By contrast, following [2] we use the terms ringlet and (bi)modulet respectively for an 'associative ring not necessarily with unity' and for a 'not necessarily unital (bi)module' over (a) ringlet(s). The term ideal (unqualified by 'left' or 'right') always means two-sided ideal. A prime ideal $P$ of a ringlet $R$ satisfies $P \neq R$. A principal ideal ring (PIR) is a ring in which every (two-sided) ideal is (two-sided) principal.

As in [22], we say that a bimodulet or ringlet is bi-noetherian or satisfies the bi-a.c.c (or some similar expression) if it satisfies the ascending chain condition on subbimodulets or on ideals respectively. Obvious terms such as bi-artinian and satisfies the bi-d.c.c are defined analogously. We let $G(R)$ denote the Brown-McCoy radical of a ringlet $R$. A ringlet or bimodulet is simple if it is non-zero and 0 is its only proper ideal or subbimodulet. A ring is polysimple if it is a finite direct sum of simple rings, and is pre-simple (respectively, pre-polysimple) if $R / G(R)$ is simple (polysimple). If $M$ is an $S-R$ bimodulet, we shall occasionally say that $M$ is $b i$-uniform, or that a subbimodulet $B$ is bi-essential in order to emphasise that we are interested in $M$ or $B$ as two-sided (sub)-object. The bi-socle of a bimodulet is the sum of its simple subbimodulets.

If $S, R$ are ringlets then an element $u$ of an $S-R$ bimodulet $B$ is normalising if $S u=u R$, and an element $v$ of an $R-R$ bimodulet $M$ is centralising if $r v=v r$ for each $r \in R$. In an $R-R$ bimodule $M$ over a ring $R$ a sequence $c_{1}, c_{2}, \ldots$ is subcentralising if $c_{1}$ is centralising in $M, c_{2}+M /\left(c_{1} R\right)$ is centralising in $M /\left(c_{1} R\right)$, and so on. In an $S-R$ bimodule $B$ over rings $S, R$ a sequence $u_{1}, u_{2}, \ldots$ is subnormalising if $u_{1}$ is normalising in $M, u_{2}+M /\left(u_{1} R\right)$ is normalising in $M /\left(u_{1} R\right)$, and so on. As in [23, 24,25 ] we call sequences of these types subcentralising and subnormalising respectively, for reasons explained in [23] and [24], though other terminologies are widely used.

We then call a ring or bimodule polycentral $(P C)$ or polynormal $(P N)$ if each principal ideal or subbimodule is generated (respectively) by a finite subcentralising or subnormalising sequence. Thus a bi-noetherian polycentral (BPC) ring or bimodule is 
one in which every ideal or subbimodule is generated by a finite subcentralising sequence, and a bi-noetherian polynormal (BPN) ring or bimodule is defined analogously (as in the Introduction). Clearly a polycentral bimodule or ring is polynormal, and a BPC bimodule or ring is BPN. It is well-known that these inclusions are strict.

This terminology is significantly different from that we have used previously, for example in [24] and [25]; the change is intended to bring our terminology largely but not completely into line with the most commonly (though not universally) used vocabulary, as deployed for example in $[\mathbf{1 8}, \mathbf{1 2}]$. There is no way of avoiding a slight incompatibility of terminology, because a significant part of our methods and results (mainly in [27]) covers rings which are $\mathrm{PC}$ or $\mathrm{PN}$ but not bi-noetherian, while all other studies we know of cover only examples which are at least bi-noetherian, and usually left-right noetherian. Unfortunately, the acronym BPC which we use here was used in a different sense in [24] and [25]; we believe the advantages of the change outweigh any temporary confusion that may be caused.

We frequently use, without specific reference, various standard facts about endomorphism rings of infinite dimensional vector spaces. The general references for this material are [8, Chapter 10] and [30].

Notation is standard with a handful of exceptions. If $R$ is a ringlet and $X$ is a non-empty subset of a left (or bi)modulet over $R$, then $L A(X)$ or $L A_{R}(X)$ or small variations thereof will denote the left annihilator in $R$ of $X$, and the symbol $R A(X)$ and its variants are analogously defined on the right. The symbols $\mathcal{I}(R)$ and spec $(R)$ denote the lattices of ideals and of prime ideals of $R$. If $R$ is a ringlet, then $P(R)$ denotes its prime radical, which is nilpotent if $R$ is bi-noetherian, and $J(R)$ denotes the Jacobson radical. Morphisms of one-sided modulets are generally written on the opposite side; ring and bimodulet morphisms are generally-except where inconvenient-written on the right. The symbol $\subset$ denotes strict inclusion.

\section{EXAMPLES}

1. Any commutative ring is a $P C$ and $P N$ ring, and is $B P C$ and $B P N$ if it is noetherian.

2. The universal enveloping algebras of finite dimensional nilpotent Lie algebras over fields of characteristic 0 are BPC rings [17], as are certain rings of fractions constructed from them [20]. Suitable group rings are BPC rings, as again are certain rings of fractions constructed from them [20]. The universal enveloping algebra of a finite dimensional solvable Lie algebra over an algebraically closed field of characteristic 0 is a BPN ring [17]. All these examples are left-right noetherian.

3. Any simple ring is a BPC ring, as more generally is any ring in which every ideal is generated by a central element. Any ring in which every ideal is generated by a normalising element is a BPN ring. Both the latter classes are studied in [22]. 
4. Clearly, each of the classes of $P C$ and of $P N$, and of BPC and of BPN rings, is closed under finite direct sums and epimorphic images.

5. A ring $R$ is $\mathrm{PC}$ if and only if each full matrix ring $M_{n}(R)$ is $\mathrm{PC}$, and is $\mathrm{BPC}$ if and only if each $M_{n}(R)$ is BPC [27]. Thus the PC and BPC properties are Morita invariant.

6. If $R$ is a PN ring then so are each full matrix ring $M_{n}(R)$, and each ring $U T_{n}(R)$ and $L T_{n}(R)$ of upper and lower triangular matrices over $R$. If $R$ is BPN then so are each $M_{n}(R), U T_{n}(R)$ and $L T_{n}(R)[27]$.

7. In [5] the authors show that, for each $n \in N$, there is a left-right noetherian prime domain $D_{n}$ with exactly $n$ prime ideals $P_{1} \subset P_{2} \subset \ldots \subset P_{n}$. Each $P_{j+1} / P_{j}$ is generated by a central element of $D_{n} / P_{j}$, with $1 \leqslant j<n$, so by Theorem $5.1, D_{n}$ is BPC. Since a commutative noetherian ring with a chain $P_{1} \subset P_{2} \subset P_{3}$ of prime ideals has infinitely many primes, this is a striking contrast with the commutative case.

For the next four examples, we fix the following notation: $R$ is a ring and $\mathcal{A}$ is a set (not necessarily finite) of $R$-automorphisms such that $\alpha \beta \alpha^{-1} \beta^{-1}$ is inner for any $\alpha, \beta \in \mathcal{A}$, and $\mathcal{T}, \mathcal{V}$ are respectively a skew polynomial and a skew Laurent extension ring of $R$, generated by indeterminates $X^{w}(w \in W)$ each of which 'skews' $R$ by an element of $\mathcal{A}$. The proofs of all statements can be found in [27].

8. Suppose all the elements of $\mathcal{A}$ are inner, and the number of indeterminates is finite. Then the ring $R$ is BPC if and only if $\mathcal{T}$ is BPC if and only if $\mathcal{V}$ is BPC.

9. Suppose all the elements of $\mathcal{A}$ are inner, and $R$ is BPC. Then $\mathcal{T}$ and $\mathcal{V}$ are polycentral rings.

10. Suppose the number of indeterminates is finite. Then $\mathcal{T}$ is BPN if and only if $\mathcal{V}$ is BPN if and only if $R$ is BPN and satisfies a further condition (*) identified in $[27]$.

11. Suppose $R$ is BPN and satisifies the condition $(\star)$. Then $\mathcal{T}$ and $\mathcal{V}$ are polynormal rings.

12. If $R$ is a BPC or a BPN ring and $\delta$ is an $R$-derivation, the skew polynomial ring $R[X ; \delta]$ is $\mathrm{BPC}$ or BPN provided that in each case a condition identified in [27] is also satisfied.

13. If $R$ is a BPC or a BPN ring, and $T$ is a ring extension of $R$ generated by an element which normalises $R$ (but is not necessarily regular in $T$, so that $T$ need not be an epimorphic image of a skew polynomial ring with one indeterminate and one automorphism), then $T$ is a BPC or a BPN ring provided that in each case a condition identified in [27] is satisfied. (This result will be applied to graded BPC: and BPN rings in the sequel [28].)

14. If $R$ is a ring and is not BPC or not BPN, then by Zorn's Lemma there exist ideals $Q, P$ maximal respectively with respect to not being generated by a finite 
subcentralising and a finite subnormalising sequence. By Theorem 5.1 the factor rings $R / Q$ and $R / P$ are prime $\mathrm{BPC}$ and $\mathrm{BPN}$ rings respectively. Thus a ring is BPN (respectively, BPC) if and only if every prime ideal is generated by a finite subnormalising (respectively, subcentralising) sequence.

15. If $R$ is a ring, and $A, B, C, D \in \mathcal{I}(R)$ are such that $R / A, R / B$ are $\mathrm{BPC}$ rings or $R / C, R / D$ are $\mathrm{BPN}$ rings then the subdirect products $R /(A \cap B), R /(C \cap D)$ are BPC and BPN respectively (Proposition 4.4). Combined with Paragraph 14 this observation gives a plentiful supply of semiprime BPC and BPN rings of finite bimodule uniform dimension. It can be shown [25] that such a ring contains a left-right Ore set of regular elements with respect to which its quotient ring is a polysimple ring. Moveover, in [25] we use this construction to define a reduced rank function for BPN bimodules, which can be used to establish various 'good' properties of BPN rings, such as a Principal Ideal Theorem-stating that a prime $P$ minimal over a normalising element has height not exceeding $1-$ and the d.c.c. on prime ideals.

16. In [26] we introduce $G$-functors, which are functors on the category of rings to itself, defined for each cardinal number $t$ (finite or infinite), and denoted by $G_{t}(-)$. There is always a natural ring inclusion $R \rightarrow G_{t}(R)$, and for $t>1$ the ring extension $R \subset G=G_{t}(R)$ has the pleasing properties that extension and contraction induce lattice isomorphisms $\mathcal{I}(R) \leftrightarrow \mathcal{I}(G)$ which respect multiplication, but that $G$ satisfies neither Goldie condition on either side. It can easily be shown [26] that a ring $R$ is BPC if and only if each $G_{t}(R)$ is BPC $(t>1)$, and that if $R$ is BPN then so is each $G_{t}(R)$.

17. Still with $G$-functors, if the cardinals $a, b$ are large enough (relative to a cardinal $d$ determined by the structure of $R$ ) then $a \neq b$ implies $G_{a}(R)$ and $G_{b}(R)$ are non-isomporphic rings. It follows that, if $R$ is a BPC or a BPN ring then, for any cardinal number $w$ whatever, the class $\left\{G_{t}(R)\right\}$ contains $w$ pairwise non-isomorphic non-noetherian $\mathrm{BPC}$ or $\mathrm{BPN}$ rings. That is, 'almost all' $\mathrm{BPC}$ and $\mathrm{BPN}$ rings are non-noetherian.

18. If $R$ is a semiprime ring with an essential ideal which is BPC or BPN as bimodule then $R$ is a BPC or a BPN ring respectively (Section 10).

\section{Bimodule categories}

We begin with an omnibus technical lemma.

Proposition 4.1. Let $B$ be a bimodule over rings $S, R$.

(i) If $u \in B$ is normalising and $C \subseteq S u=u R$ is a subbimodule of $B$ then there exist ideals $Y, X$ of $S, R$ respectively such that $C=Y u=u X$.

(ii) If $B$ is generated by a finite subnormalising sequence, and if one of $S, R$ is bi-noetherian or bi-artinian then $B$ has the same property. 
(iii) If $B$ is a simple bimodule then $L A(B)=L A(u)$ and $R A(B)=R A(u)$ are maximal ideals of $S, R$ respectively.

(iv) If $B=S u=u R$ is left-right faithful then the map $S \rightarrow R$ defined by $s u=u r$ for all $s \in S$ is a ring isomorphism.

Proof: Each of (i), (iii) and (iv) is straightforward, and (ii) follows easily from (i) using the isomorphism theorems and induction.

A simple bimodulet $B$ over ringlets $S, R$ is said to be supersimple if $B=S u=u R$ for some $u \in B$. If $B$ is a bimodule then is satisfies part (iii) of the previous proposition, so by part (iv) $S / L A(B)$ and $R / R A(B)$ are isomorphic simple rings. There is a connection between supersimple bimodules (over rings) and a result (closely resembling Nakayama's Lemma) concerning polynormal or other 'nicely'-generated bimodules:

LemMa 4.2. Let $S, R$ be rings and $B$ be an $S-R$ bimodule with a maximal subbimodule $M$ (for example, a $B P N$ bimodule). Suppose that $B$ is either $P N$ or generated by a finite subnormalising sequence, and that $H, G$ are the Brown-McCoy radicals of $S, R$ respectively. Then $H B+B G \subseteq M$.

Proof: This follows from Proposition 4.1 (iii), since $B / M$ is obviously supersimple.

Lemma 4.3. Let $S, R$ be rings and $0 \rightarrow M^{\prime} \rightarrow M \rightarrow M^{\prime \prime} \rightarrow 0$ be an exact sequence of $S-R$ bimodules. Then:

(i) if $M^{\prime \prime}$ is $P N$ and $M^{\prime}$ is $B P N$ then $M$ is $P N$, and is $B P N$ when $M$ (equivalently $M^{\prime \prime}$ ) is bi-noetherian;

(ii) if $S=R, M^{\prime \prime}$ is $P C$ and $M^{\prime}$ is $B P C$, then $M$ is $P C$, and is $B P C$ when $M$ (equivalently, $M^{\prime \prime}$ ) is bi-noetherian.

Proof: This is an easy consequence of the isomorphism theorems.

Proposition 4.4. Let $R$ be a ring and $A, B \in \mathcal{I}(R)$. Suppose that $R / A$ and $R / B$ are $B P N$ (or $B P C$ ). Then so are $(R / A) \oplus(R / B)$ and $R /(A \cap B)$.

Proof: The first assertion is obvious. For the second, note that $(A+B) / B \simeq$ $A /(A \cap B)$ as $R-R$ bimodule, so $A /(A \cap B)$ is a BPN (BPC) bimodule. Applying Lemma 4.3 to the exact sequence $0 \rightarrow A /(A \cap B) \rightarrow R /(A \cap B) \rightarrow R / A \rightarrow 0$, the result follows.

Remark. In the circumstances of the previous proposition, $R / A B$ can fail to be a BPN (or BPC) ring. For example, let $K \subset F$ be commutative fields, and let $R$ be the ring $\left(\begin{array}{cc}K & F \\ 0 & F\end{array}\right)$ and $P$ the ideal $\left(\begin{array}{cc}0 & F \\ 0 & 0\end{array}\right)$. Then $R / P$ is a $\mathrm{BPC}$ ring, but $P^{2}=0$ so $R=R / P^{2}$ is not a BPN ring. 
Theorem 4.5. Let $S, R$ be rings and $B, M$ be an $S-R$ bimodule and an $R-R$ bimodule respectively. Suppose $B$ is generated by a finite subnormalising sequence and $M$ by a finite subcentralising sequence. Then:

(i) if $R$ is a $B P C$ ring then $M$ is a $B P C$ bimodule;

(ii) if one of $S, R$ is a $B P N$ ring then $B$ is a $B P N$ bimodule.

Proof: We prove (i); the proof of (ii) is almost identical. Let $N$ be a subbimodule of $M$, and suppose that $c_{1}, \ldots, c_{n}$ is a finite subcentralising sequence that generates $M$. If $n=1$ then the result is immediate from Proposition 4.1(i), so suppose $n>1$. Let $E$ be the subbimodule generated by $c_{1}, \ldots, c_{n-1}$. By the obvious inductive hypothesis $N^{\prime}=N \cap E$ is $\mathrm{BPC}$, and by the $n=1$ case $N / N^{\prime} \simeq(N+E) / E \subseteq M / E$ is generated by a subcentralising sequence. Taking suitable inverse images in $N$, and a sequence generating $N^{\prime}$, we obtain a subcentralising sequence generating $N$.

Corollary 4.6. Let $S, R$ be rings and $\mathrm{B}, \mathrm{M}$ be respectively the categories of $S-R$ bimodules generated by finite subnormalising sequences and $R-R$ bimodules generated by finite subcentralising sequences. Then:

(i) if $R$ is a $B P C$ ring then $\mathrm{M}$ is an abelian category;

(ii) if one of $S, R$ is a BPN ring then $\mathbf{B}$ is an abelian category.

ProOF: Closure under finite direct sums follows from the fact that a finite sum of bimodules generated by appropriate sequences is similarly generated (putting the sequences themselves in sequence), and closure under subbimodules is given by the theorem.

Theorem 4.5 is essentially a consequence of the isomorphism theorems, but it has an interesting converse. We proceed via an easy special case to show that, supposing only the existence of a left-right faithful BPC $R-R$ bimodule or a right faithful BPN $S-R$ bimodule, the ring $R$ must be BPC or BPN (respectively). The obvious analogue of this result for left-right noetherian rings is, that if $B$ is a left-right faithful left-right noetherian $R-R$ bimodule, then $R$ is a left-right noetherian ring. In Section 10 we make other comparisions between BPC, BPN, left-right noetherian and left-right artinian rings.

We begin with a technical definition: an $S-R$ bimodule $B$ is $N$-cyclic if $B=$ $S u=u R$ for some $u \in B$, and an $R-R$ bimodule $M$ is C.cyclic if $M=R v=v R$ for some centralising element $v \in M$.

LEMMA 4.7. Let $B$ be a right faithful $S-R$ bimodule, and suppose that:

(i) $B$ is $B P N$ and is a finite sum of $N$-cyclic modules; or

(ii) $S=R$, and $B$ is $B P C$ and is a finite sum of $C$-cyclic bimodules. 
Then $R$ as ring is, respectively, BPN or BPC.

Proof: We prove case (i) only; case (ii) is effectively a special case.

Suppose first that $B$ is $\mathrm{N}$-cyclic, and that $B=S v=v R$. If $A \in \mathcal{I}(R)$ suppose that the bimodule $v A$ is generated by the $S-R$ subnormalising sequence $v r_{1}, \ldots v r_{n}$ for $r_{i} \in R$. Then since $R A(B)=R A(v)=0, r_{i} \in A$ for each $i$, and it is easy to see that $r_{1}, \ldots, r_{n}$ is an $R-R$ subnormalising sequence generating $A$.

So suppose that $v_{1}, \ldots v_{t}$ are $S-R$ normalising elements of $B$ such that $B=$ $\sum v_{j} R$. Let $A_{j}=R A\left(v_{j}\right)=R A\left(v_{j} R\right)$ for $1 \leqslant j \leqslant t$. Then each $A_{j}$ is an ideal of $R$, and $\bigcap_{j=1}^{t} A_{j}=0$. Suppose $B$ is BPN. From above each $R / A_{j}$ is a BPN ring, so by Proposition 4.4 (and induction) $R$ is a BPN ring.

We define some necessary notation before the next result. Suppose that $B$ is a bimodule over rings $S, R$, and that $B$ is generated by a finite $S-R$ subnormalising sequence $u_{1}, \ldots, u_{n}$. Let $W_{0}=0$, and $W_{j}=u_{1} R+\ldots+u_{j} R=S u_{1}+\ldots+S u_{j}$ for $1 \leqslant j \leqslant n$. Let $A=R A_{R}\left(W_{1}\right), X=R A_{R}\left(B / W_{1}\right)$ and $Y=R A_{R}\left(W_{n-1}\right)$. If $p \in N$, let $B p=B \oplus \ldots \oplus B$ ( $p$ copies).

TheOREM 4.8. Let $B$ be a right faithful $S-R$ bimodule,and suppose that

(i) $B$ is $B P N$, or

(ii) $S=R$ and $B$ is $B P C$.

Then $R$ as ring is, respectively, BPN or BPC.

Proof: Again we prove (i), regarding (ii) as a special case. Suppose that $B$ is generated by the finite subnormalising sequence $u_{1}, \ldots, u_{n}$. By Lemma 4.7 we may suppose that $n>1$ and that the result is true for every BPN $S-R$ bimodule generated by an $S-R$ subnormalising sequence of $r \leqslant n-1$ terms. Thus (in the notation just defined) $R / X$ and $R / Y$ are BPN rings. By Proposition $4.4 R /(X \cap Y)$ is a BPN ring. By Lemma 4.3 it therefore suffices to show that $T=X \cap Y$ is a BPN $R-R$ bimodule.

Let $f: R \rightarrow B^{n}$ be the map $x \rightarrow\left(u_{1} x, u_{2} x, \ldots, u_{n} x\right)$. Then $f$ is a right $R$ module embedding of $R$ in $B^{n}$. Suppose $x \in T$. Then $u_{j} x=0$ for $1 \leqslant j<n$, and $u_{n} x=u_{1} y(x)$ for some $y(x) \in R$, so $f(x)=\left(0, \ldots, 0, u_{1} y(x)\right)$. Let $V \in \mathcal{I}(R)$, $V \subseteq T$. If $x \in V, s \in S$ then there exist $t_{i} \in R$ such that $s u_{1} y(x)=s u_{n} x=$ $\sum_{i=1}^{n} u_{i} t_{i} x=u_{n} t_{n} x=u_{1} y\left(t_{n} x\right)$, since $x \in V \subseteq T$. Hence $f(V)$ is a subbimodule of $B^{n}$, and in particular $f(T)$ is a subbimodule. Let $U \subseteq f(T)$ be a subbimodule of $B^{n}$, so that $f^{-1}(U)$ is a right submodule of $T$. Suppose $m \in f^{-1}(U)$ and $r \in R$. Then there exist $s_{i} \in S$ such that

$$
u_{n} r m=\sum_{i=1}^{n} s_{i} u_{i} m=s_{n} u_{1} y(m)
$$


Since $u_{1} y(m) \in U$ it follows that $f(r m) \in U$, so $f^{-1}(U)$ is an ideal of $R$.

Let $\Pi: B^{n} \rightarrow B$ be the projection on the $n$th coordinate, and $\alpha: u_{1} R \rightarrow R / A$ be the map $\alpha\left(u_{1} x\right)=(x+A)$. Then the composite map $g=\alpha \Pi f: T \rightarrow R / A$ is a right $R$-module embedding which induces a lattice isomorphism of the $R-R$ subbimodules of $T$ and $g(T)$. Let $V \in \mathcal{I}(R),{ }^{\prime} V \subseteq T$. By Lemma 4.7, $R / A$ is a BPN $R-$ $R$ bimodule. Suppose that $g(V)$ is generated by the finite subnormalising sequence $x_{1}, \ldots, x_{t}$, and let $\tilde{x}_{j}, y_{j}$ be such that, for $1 \leqslant j \leqslant t, y_{j}=g^{-1}\left(x_{j}\right)$ (so that each $y_{j}$ is unique) and $\alpha\left(u_{1} \tilde{x}_{j}\right)=x_{j}$. If $a \in R$ then there exist $a^{\prime} \in R, s \in S$ such that $g\left(y_{1} a\right)=x_{1} a=\alpha\left(u_{1} \tilde{x}_{1} a\right)=\alpha\left(s u_{1} \tilde{x}_{1}\right)=g\left(a^{\prime} y_{1}\right)$. Hence $a^{\prime} y_{1}=y_{1} a$. The argument is reversible, so $y_{1}$ is $R-R$ normalising. Iterating, it turns out that $y_{1}, \ldots, y_{t}$ is an $R-R$ subnormalising sequence; to avoid notational clutter, we show only that $y_{1} R+y_{2} R=R y_{1}+R y_{2}$. If $b \in R$ then there exist $b^{\prime}, b^{\prime \prime} \in S, c^{\prime}, c^{\prime \prime} \in R$ such that $g\left(y_{2} b\right)=x_{2} b=\alpha\left(b^{\prime} u_{1} \tilde{x}_{1}\right)+\alpha\left(b^{\prime \prime} u_{1} \tilde{x}_{2}\right)=g\left(c^{\prime} y_{1}\right)+g\left(c^{\prime \prime} y_{2}\right)$, so $y_{1} R+y_{2} R \subseteq R y_{1}+R y_{2}$. Here too the argument is reversible, to give equality. Thus $T$ is a BPN $R-R$ bimodule, so $R$ is a BPN ring.

The assumption that $B$ is right faithful is obviously necessary for Theorem 4.8, since for any ring $R$ and maximal ideal $M$ of $R$ the simple ring $R / M$ is BPC.

The same argument can be adapted to prove the following result, which is needed in Section 10.

Proposition 4.9. Suppose that $B$ is a right faithful BPC $R-R$ bimodule. Then $B$ is left faithful.

Proof (SKETCH): Using the notation of the proof of the theorem, and letting $Q=L A(M)$, it turns out that $Q \neq 0$ implies that $g(Q)$ is a non-zero ideal of $R / A$, contained in $(T+A) / A$. But this is impossible, since $L A\left(u_{1}\right)=R A\left(u_{1}\right)$, and $\mathcal{I}(R)$ is a (bi-)noetherian lattice.

Example. A left-right artinian ring $R$ and a right faithful, left-right artinian BPN $R-R$ bimodule $M$ which is not left faithful.

Let $K$ be a commutative field, and $R$ be the commutative $K$-algebra of dimension 3 over $K$ generated by elements $x, y$ such that $x^{2}=y^{2}=x y=0$. Let $\alpha$ be the $K$-algebra automorphism which interchanges $x$ and $y$. Let $B$ be the $R-R$ bimodule $R / y R$, and $N$ be the $R-R$ bimodule on $R / x R$ in which right multiplication is defined by the natural map $\pi: R \rightarrow R / x R$ and left multiplication by the composite map $\alpha \pi$ ( $\alpha$ first). Then $M=B \oplus N$ has the required properties.

Thus the analogue of Proposition 4.9 for BPN bimodules is false. It is true in the case of N-cyclic BPN bimodules, but not (as the example shows) for a sum of two or more of them. 


\section{PRime AND MAXIMAL IDEals}

Many results of the previous section correspond to statements about commutative noetherian rings and their finitely-generated modules which appear to go back to E. Noether. They provide the key step in the proof of the following results, which extend the characterisation of noetherian commutative rings due to I.S. Cohen [6, Theorem 2].

Theorem 5.1. Let $R$ be a ring and $Q, P \in \mathcal{I}(R)$. Suppose that $Q, P$ are maximal with respect to not being generated by (respectively) a finitely subcentralising and a finite subnormalising sequence. Then $Q, P$ are prime ideals of $R$. Hence a ring is $B P C(B P N)$ if and only if every prime ideal is generated by a finite subcentralising (subnormalising) sequence.

ProOF: We prove the subcentralising case; the proof of the other case is virtually identical. Suppose $Q$ is as described and not prime. Then $Q \neq R$ and there exist $A$, $B \in \mathcal{I}(R)$ such that $Q \subset A, Q \subset B$ and $A B \subseteq Q$. If $A \cap B=Q$ then $A B+B A \subseteq Q$ and $A /(A B+B A)$ is an $(R / Q)-(R / Q)$ bimodule generated by a finite subcentralising sequence. Since $R / Q$ is a BPC ring, $A /(A B+B A)$ is a BPC $R-R$ bimodule, so $Q /(A B+B A)$ is generated by a finite $R-R$ subcentralising sequence. But it is easy to check that $A B+B A$ is also so generated, hence $Q$ is; contradiction. In the case $Q \subset(A \cap B)$, let $A \cap B=C$; we have $C^{2} \subseteq P$ and $C / C^{2}$ turns out similarly to be a BPC $R-R$ bimodule; yielding a similar contradiction.

For the final statements, note that an ideal not generated by a finite subcentralising (subnormalising) sequence can be expanded to a maximal one by Zorn's Lemma.

In [22] we studied rings in which every ideal was generated by a normalising or by a central element, and which we called (respectively) quasi-commutative principal ideal rings (QCPIRs) and strongly quasi-commutative principal ideal rings (SQCPIRs). We showed that a ring is a QCPIR or an SQCPIR if and only if every prime ideal is generated by a normalising or a central element respectively, just like the corresponding results for $B P C$ and $B P N$ rings proved above. There is no analogous characterisation of left-right noetherian or left-right artinian rings (Remark 7, Section 10).

Since every QCPIR is a BPN ring, and every SQCPIR is a BPC ring, it is natural to enquire under what conditions a BPN or a BPC ring is a QCPIR or an SQCPIR. The solution to this problem is given by Theorem 5.3 below.

LEMMA 5.2. If $R$ is a $B P N$ or a right noetherian ring and $R q=q R$ is a maximal ideal with no idempotent power then $P=\bigcap_{n=1}^{\infty} R q^{n}$ is a minimal prime of $R$.

Proof: That $P$ is prime and that $P=P q=q P$ follows from [22, Lemma 3.4]. If $P$ is not minimal, let $A \subset P$ be prime; by passing to the factor $\operatorname{ring} R / A$ we 
may assume that $R$ is prime, $P \neq 0$ and $q$ is regular. Now $P$ contains a non-zero (hence regular) normalising element (if $R$ is BPN) or a regular element (if $R$ is right noetherian). In either case, denote this element by $u$. Since $P=P q$, we can solve the system of equations:

$$
u=u_{0}=u_{1} q=\ldots=u_{n} q^{n}=\ldots
$$

for $u_{j} \in P$. Since $q$ is regular, each $u_{j}$ is either normalising or regular, and hence is certainly regular. But by either bi-a.c.c. or right a.c.c., there exists $j \in N$ such that $u_{j} R=u_{j+1} R=\ldots$. Hence $u_{j+1}=u_{j} r$ for some $r \in R$. But $u_{j}=u_{j+1} q$, so $u_{j+1}(1-q r)=0$, which by the regularity of $u_{j+1}$ implies $q R=R$; contradiction.

TheOREM 5.3. Let $R$ be a ring, and suppose that $R$ is BPN or noetherian. Then $R$ is a $Q C P I R$ (respectively, an SQCPIR) if and only if every maximal ideal of $R$ is generated by a normalising (central) element.

Proof: We prove the normalising case; the proof of the other is virtually identical.

One implication is trivial, so we may assume that $R$ is a BPN or a right noetherian ring in which every maximal ideal is generated by a normalising element. By $[22$, Lemma 3.4 and Proposition 4.1] we may suppose no maximal ideal has an idempotent power, and then, by the previous lemma, that each maximal ideal is of height 1 . It suffices to show that $R$ is semiprime, because distinct minimal primes are clearly comaximal, and the result then follows from the structure theorems in [22]. So suppose that the prime radical $P=P(R)$ of $R$ is non-zero. Let $K \subset P$ be an ideal of $R$ such that $P / K$ is a simple, and hence supersimple, bimodule. By Proposition 4.1 $X=L A_{R / K}(P / K)$ is a maximal ideal of $R / K$. But the prime ideals of $R / K$ are simply the ideals $Q / K$ where $Q$ is a prime of $R$, and by [22, Proposition 3.6] $X$ is generated by a regular normalising element of $R / K$; contradiction. Hence $P=0$, as required.

The commutative case of this result is due to Kaplansky [13, p.486]. Neither it nor Lemma 5.2 can, however, be extended to the case of an arbitary bi-noetherian ring, or even to principal ideal rings. Thus in [22] we give examples, for each $n \in N$, of prime PIRs in which every maximal ideal is of height $n+1$, but is generated by a central element.

The other obvious question to ask is: under what conditions must a BPN ring be a BPC ring? The following hypothesis seems highly plausible, given what has gone before, but we have been unable to decide whether it is true or false.

CONJECTURE 5.4. A BPN ring $R$ is a BPC ring if and only if every maximal ideal of $R$ is generated by a finite subcentralising sequence. 


\section{BI-ARTINIAN RINGS}

It is well-known (for example; see $[1,6]$ ) that a commutative ring satisfies the (bi)d.c.c. if and only if it is (bi)-noetherian and every prime ideal is maximal. A similar, though not identical, result is true for polynormal rings.

Theorem 6.1. Let $R$ be a polynormal ring.

(a) Suppose that $R$ is bi-artinian, and that either

(i) $R$ is bi-noetherian, or

(ii) there exists an integer $k \in N$ such that every principal ideal of $R$ is generated by a subnormalising sequence of at most $k$ terms;

then every prime ideal of $R$ is maximal, and $R$ has a bimodule composition series (so is $B P N$ ).

(b) Suppose that $R$ is bi-noetherian (hence $B P N$ ) and every prime ideal of $R$ is maximal; then $R$ is bi-artinian (so has a bimodule composition series).

Proof: (a) Suppose that $R$ is a bi-artinian ring. There exists a minimal finite intersection $G$ of maximal ideals of $R$, and clearly the only maximal ideals of $R$ are those which contain $G$, so $G$ is the Brown-McCoy radical of $R$. By bi-d.c.c. $G$ has an idempotent power, and it is easy to check that, by Lemma 4.2, this must be zero. It follows that the zero ideal of $R$ is a product of maximal ideals, so every prime is maximal.

If (i) $R$ is bi-noetherian then it has a bimodule composition series. So suppose (ii) is satisfied, so that we do not know that $R$ is bi-noetherian. Let $M$ be one of the finitely many non-zero bimodules of form $G^{i} / G^{i+1}$ where $i \in \mathrm{N}$. The ring $S=R / G$ is polysimple, and $M$ is naturally an $S-S$ bimodule. From (ii) we can find a finite sequence of bimodules: $0=W_{0} \subseteq W_{1} \subseteq \ldots \subseteq W_{r}=M$, such that $r \leqslant k$ and each $V_{j}=W_{j+1} / W_{j}(0 \leqslant j<r)$ is generated as $S-S$ bimodule by normalising elements. Since $S$ is a direct sum of supersimple bimodules so is each $V_{j}$. But $R$ can only be bi-artinian provided that each $V_{j}$ is bi-artinian, and that happens if and only if each $V_{j}$ is bi-noetherian, and in particular is finitely-generated. Putting the pieces together, $R$ is bi-noetherian, so it has a bimodule composition series.

Suppose, conversely, that $R$ is a BPN ring and every prime ideal of $R$ is maximal. By bi-a.c.c. the zero ideal is a finite product of prime, hence of maximal, ideals. Thus $R$ has finitely many maximal ideals, and their intersection $G$ is nilpotent. But $T=R / G$ is then polysimple, hence bi-artinian, and each of the finitely many non-zero bimodules $G^{i} / G^{i+1}$ is bi-artinian by Proposition 4.1. Hence $R$ is bi-artinian (and the bimodule length of $R$ is an upper bound for the length of any principal ideal).

We say that a ring $R$ satisfies the restricted bi-d.c.c. if $R$ is not bi-artinian but $R / A$ 
is bi-artinian for any non-zero ideal $A$ (the definition used by Cohen for commutative rings in [6]). The following result then follows using Theorem 6.1 almost exactly as in the commutative case.

TheOREM 6.2. A BPN ring $R$ satisfies the restricted bi-d.c.c if and only if it is prime and every non-zero prime ideal is maximal.

The analogy with bi-artinian commutative rings is not complete in two respects. First, the case of the $n \times n$ upper triangular matrices $(n>1)$ over a field shows that an indecomposable bi-artinian BPN ring need not be pre-simple (whilst an indecomposable bi-artinian commutative ring must be local). Second, there is an example [24, Example 3.5.5] of a polycentral ring which is bi-artinian (and hence has nilpotent Brown-McCoy radical) but is not bi-noetherian. It contains principal ideals with arbitarily long bimodule composition series.

On the other hand, the contrast with left-right noetherian rings is striking: in [19] Robson exhibits a noetherian domain with exactly three ideals; this ring has a bimodule composition series but an idempotent non-zero Brown-McCoy radical.

\section{THE ARTINIAN AND BI-ARTINIAN RADICALS}

We recall from [3] and [4] that, if $R$ is a left-right noetherian ring then $I \in \mathcal{I}(R)$ is left artinian if and only if it is right artinian. The unique largest right artinian right ideal is a two-sided ideal called the artinian radical, and is (therefore) also the unique largest left artinian left ideal. Moreover, if $A(R)$ is the artinian radical of the noetherian ring $R$, then $A /(R / A(R))=0$ and $A(R)$ has various other pleasing properties, such as (a) $A(R)$ is a right complement right ideal, and (b) if $A(R)$ is right essential then $R$ is right artinian. The artinian radical can be used (see [3] and [4]) to provide elegant proofs of various (previously known) results about noetherian rings.

We show in this section that there are two very similar radicals $A(R) \subseteq B(R)$ for a BPN ring $R$, and that they may similarly be used to prove various new results about BPN rings (Section 9). Our starting point is the following:

Theorem 7.1. Let $B$ be a $B P N$ bimodule over rings $S, R$. Then:

(i) $S^{B}$ is left artinian if and only if $B_{R}$ is right artinian;

(ii) $B_{R}$ is right artinian if and only if $R /(R A(B))$ is right artinian;

(iii) $B$ is bi-artinian if and only if $R / R A(B)$ is bi-artinian.

Proof: (i) Suppose that ${ }_{S} B$ is left artinian. Then it has a bimodule composition series each simple factor of which is supersimple. The result for supersimple bimodules follows from that for simple rings, and that for $B$ then follows by induction.

(ii) Since $B$ is right finitely-generated, one way round this is obvious. Since $B$ is 
left finitely-generated, $(R / R A(B))_{R}$ embeds as right $R$-module in a finite direct sum $\left(B_{R}\right)^{n}$ of copies of $B_{R}$, and $\left(B_{R}\right)^{n}$ is right artinian when $B_{R}$ is.

(iii) If $R / R A(B)$ is bi-artinian this follows by Proposition 4.1(ii).

So suppose that $B$ is bi-artinian. By Theorem $4.8 R / R A(B)$ is a BPN ring, so by Theorem 6.2 we need only show that, if $B$ has a bimodule compostion series and is right faithful then every $P \in \operatorname{spec}(R)$ is maximal. If $B$ has a bimodule composition series then each factor is supersimple. By Proposition 4.1(iii), the right annihilator of each factor is a maximal ideal. Let the intersection of the finitely many maximal ideals involved be $T$. Then the zero ideal contains a power of $T$, and hence a product of maximal ideals. It follows that every prime ideal is maximal.

COROllaRY 7.2. A BPN ring is right artinian if and only if it is left artinian.

Theorem 7.3. Let $R$ be a $B P N$ ring. Then $R$ has two ideals $A(R) \subseteq B(R)$ such that:

(i) $A(R)$ is the unique largest right artinian right ideal of $R$ and is the unique largest left artinian left ideal of $R$, and has both right and left composition series.

(ii) $B(R)$ is the unique largest ideal of $R$ to have a bimodule composition series.

Proof: (i) Since $R$ is bi-noetherian, the right socle series for $R$ becomes stationary; the stationary term is an ideal, which can be denoted by $A(R)$. If $S=S^{\star}(R)$ is the right socle then $S$ is an ideal, right fuitely-generated by elements $x_{1}, \ldots, x_{n}$. Each $x_{j}$ is contained in a finite direct sum of simple right ideals; hence $S_{R}$ is artinian semisimple. It follows that $A(R)$ has a right composition series and contains every right artinian right ideal of $R$. By Theorem $7.1 A(R)$ is left artinian, and the results follow by symmetry.

(ii) It is clear that $B(R)$ exists and that $A(R) \subseteq B(R)$.

If $R$ is a left-right noetherian BPN ring, then $A(R)$ as defined here is obviously the same ideal as the $A(R)$ defined in [3] or [4]. We can now make available the techniques of the Moss prime used in those references. The following result will allow us to 'move from one side to the other' of an ideal of a BPN ring.

LEMMA 7.4. Let $R$ be a $B P N$ ring and $I, X$ be ideals such that $R / X$ is biartinian (or right artinian). Then there is an ideal $Y$ of $R$ such that $R / Y$ is bi-artinian (respectively, right artinian) and $Y I \subseteq I X$.

Proof: By Proposition 4.1(ii) $I / I X$ has a bimodule composition series as $R$ $(R / X)$ bimodule. Let $Y=L A(I / I X)$; by the left analogue of Theorem 7.1(iii) (or of 
Theorem 7.1(ii), plus Corollary 7.2) $R / Y$ is bi-artinian (or right artinian), and back in $R, Y I \subseteq I X$.

Proposition 7.5. Let $S, R$ be rings and $R$ be $B P N$. Suppose $M$ is a $B P N$ $S-R$ bimodule such that $R /\left(R A_{R}(M)\right)$ is not bi-artinian. Let $Q, P$ be ideals of $R$ maximal with respect to the properties:

(1) $R / Q$ is not left-right artinian and $Q=R A_{R}(K)$ for some subbimodule $K$ of $M$;

(2) $R / P$ is not bi-artinian and $P=R A_{R}(N)$ for some subbimodule $N$ of $M$.

Then $Q, P$ are prime ideals of $R$.

Proof: (After [4]). Let $A, B$ be ideals of $R$ which strictly contain $P$, and suppose $A B \subseteq P$. If $P=R A(N)$ for the subbimodule $N$ of $M$ then $N A B=0$ so we can assume $B=R A_{R}(N A)$. Because $P \subset B R / B$ is bi-artinian, so $N A$ is bi-artinian. By Lemma 7.4 there is an ideal $C$ of $R$ such that $R / C$ is bi-artinian and $C A \subseteq A B$. Hence $N C A=0$. If $D=R A(N C)$ then $A \subseteq D$. Hence $R / D$ is bi-artinian, and $C / C D$ is bi-artinian by Proposition 4.1(ii), since $R / D$ is. Hence $R / C D$ is bi-artinian, so $R / P$ is; contradiction. The proof for $Q$ is similar.

By analogy with [3, Chapter 7], we call a prime of either of these types a Moss prime associated with $M$. Just which property a Moss prime is associated with will be made clear in each case.

Proposition 7.6. Let $S, R$ be rings and $R$ be BPN. Suppose that $M$ is a BPN $S-R$ bimodule, and that the bi-socle (respectively, right socle) of $M$ is bi-essential. Then $M$ is bi-artinian (respectively, right artinian).

Proof: Suppose the distinct supersimple subbimodules of $M$ are $B_{j}$, for $1 \leqslant j \leqslant$ $n$. For each $j$, let $A_{j}$ be a subbimodule of $M$ maximal with respect to the properties: $B_{j} \cap A_{j}=0$, and $i \neq j$ implies $B_{i} \subseteq A_{j}$. Then $A_{1} \cap \ldots \cap A_{n}=0$, and each $M / A_{j}$ is subdirectly irreducible. Moreover, $M$ is bi-artinian if and only if each $M / A_{j}$ is, so we may assume that $M$ is subdirectly irreducible, with heart $H$, but is not bi-artinian.

Let $P$ be a Moss prime associated with $M$, such that $R / P$ is not bi-artinian. If $N$ is the subbimodule of $M$ such that $P=R A(N)$ then $0 \neq N$ since $P \neq R$, so $H \subseteq N$ since $M$ is bi-uniform and $H$ is simple and bi-essential. Let $T=R / P$, and let $u_{1}, \ldots, u_{r}$ be the subnormalising sequence generating $N$ as $S-T$ bimodule. Let $L_{0}=0$, and $L_{j}=u_{1} T+\ldots+u_{j} T$ for $1 \leqslant j \leqslant r$. We may, without loss of generality, suppose that $L_{j-1} \subset L_{j}$ for $1 \leqslant j \leqslant r$. Now let $A_{j}=R A_{T}\left(L_{j} / L_{j-1}\right)$ for $1 \leqslant j \leqslant r$. Then $N A_{r} A_{r-1} \ldots A_{1}=0$. Since $N$ is a right faithful $T$-module and $T$ is a prime, there exists an integer $j$ such that $A_{j}=0$, and hence a least one, say $m$. 
Let $w=u_{m}$, and $X=A_{m-1} \ldots A_{1}$. Then $w X=S w X$ is a non-zero subbimodule of $M$. Now for some $v \in H, H=S v=v T$, so $v=w x$ for some $x \in X, x \neq 0$. Since $H$ is supersimple and $T$ is not bi-artinian, $V=R A_{T}(H)$ is a non-zero maximal ideal of $T$. But then

$$
w x V=w x T V=v T V=0=S v T V=(S w)(x T V)=w(T x T) V .
$$

Hence $(T x T) V \subseteq A_{m}=0$, contradicting the fact that $T$ is a prime ring. It follows that $M$ is bi-artinian.

The right artinian case is similar.

The following result is almost immediate.

Theorem 7.7. Let $R$ be a BPN ring. Then:

(i) $R$ is bi-artinian if and only if the bi-socle $B S(R)$ of $R$ is bi-essential;

(ii) $R$ is right artinian if and only if the right socle $S^{\star}(R)$ of $R$ is bi-essential.

Proof: If $R$ is bi-artinian then it has finite bimodule uniform dimension, so $B S(R)$ is bi-essential. Suppose, conversely, that $B S(R)$ is bi-essential. Then $R$ is bi-artinian by Proposition 7.6. The right artinian case is similar.

COROLlary 7.8. Let $R$ be a BPN ring. Then the following conditions on $R$ are equivalent:

(i) $R$ is pre-polysimple and the Brown-McCoy radical of $R$ is nilpotent;

(ii) $R$ is bi-artinian;

(iii) $B(R)$ is bi-essential in $R$.

There is an important special case of Theorem 7.7 which can be proved by a simplification of the above argument, but also has a noteworthy simple direct proof, independent of all the previous results of this paper.

Proposition 7.9. Let $R$ be a prime $B P N$ ring. Then $B(R) \neq 0$ if and only if $R$ is a simple ring, so a prime subdirectly irreducible BPN ring is simple. Moreover, $A(R) \neq 0$ if and only if $R$ is a left-right artinian simple ring.

FIRST Proof: Suppose $B=B(R) \neq 0$. Since $R$ is prime, $R A_{R}(B)=0$, so by Theorem 7.1(iii) $R=R / R A_{R}(B)$ is bi-artinian. Since $R$ is prime, and every prime ideal is maximal (Theorem 6.1) $R$ is simple.

SECOND PROOF: Suppose $B=B(R) \neq 0$. Since $R$ is prime, it is subdirectly irreducible, with supersimple heart $H$. It is easy to check that $H$ must contain an idempotent non-zero normalising element, which by [7, Theorem 4] is central. Since $R$ is prime, $H=R$.

With either proof, the case where $A(R) \neq 0$ then follows at once. 
Remarks. 1. There is an example due to Stafford [21] of a prime, subdirectly irreducible left-right noetherian ring $R$ which is not bi-artinian. Thus the analogues for left-right noetherian rings of the (bi-artinian parts of) the three last results are false, though a prime noetherian ring with non-zero right socle is indeed artinian simple.

2. It is also noteworthy that for a BPN bimodule $M$ or ring $R$ to be right artinian we need only assume that the right socle is bi-essential, rather than right-essential. For left-right noetherian rings this is false [3, p.64]. Among arbitary rings, there exist non-artinian prime rings with right essential right socle, for example the endomorphism rings of infinite dimensional left vector spaces.

The following result summarises the properties of $A(R)$ and $B(R)$. It bears a striking resemblance to Theorem 1.3 of [4].

Theorem 7.10. Let $R$ be a $B P N$ ring. Then:

(i) The artinian radical $A(R)$ of $R$ is a two-sided ideal which is the unique largest right artinian right ideal and also the unique largest left artinian left ideal of $R$;

(ii) $A(R / A(R))=0$, that is, $R / A$ has no non-zero one-sided artinian onesided ideals;

(iii) $A$ is a complement right ideal and a complement left ideal;

(iv) $A$ is a right annihilator and a left annihilator;

(v) $A$ is a left-right noetherian $R-R$ bimodule;

(vi) The bi-artinian radical $B(R)$ is a unique largest ideal of $R$ to have a bimodule composition series;

(vii) $B(R / B(R))=0$, that is, $R / B$ has no non-zero bi-artinian ideals;

(viii) each of $A, B$ is a complement ideal, that is, $A$ and $B$ have no proper essential extensions as ideals of $R$;

(ix) $B$ is a left annihilator and a right annihilator.

PROOF: It is clear from previous results that we need only prove (iii), (iv), (viii) and (ix).

(iii). Let $I$ be a right ideal of $R$ which contains a right essential right artinian submodule, and let $J=R I$. Since $J$ is left finitely-generated, $I$ satisfies the Gabriel $H$-condition, so there is a right embedding $R / R A(I) \rightarrow I^{n}$ for some integer $n \in \mathbb{N}$. Hence $S=R / R A(I)$ has right essential, and hence bi-essential, right socle, so $S$ is right artinian. Since $R A(I)=R A(J), J$ is a right artinian $R-S$ bimodule, so $I \subseteq J$ is right artinian. It follows that a right essential extension of a right artinian right ideal of $R$ is right artinian, and (iii) follows at once.

(iv). By Theorem $7.1, R / R A_{R}(A)$ is a left-right artinian ring. But $L A_{R}\left(R A_{R}(A)\right)$ is a BPN $R-\left(R / R A_{R}(A)\right)$ bimodule, so is left-right artinian by Theorem 7.1 
again. Hence $A \subseteq L A_{R}\left(R A_{R}(A)\right) \subseteq A$ by part (i), and then symmetry forces $A=L A_{R}\left(R A_{R}(A)\right)=R A_{R}\left(L A_{R}(A)\right)$.

(viii). Suppose $E$ is a bi-artinian ideal of a BPN ring $R$, and $I$ is an essential extension of $E$ as an ideal of $R$. Let $K$ be an ideal of $R$ maximal with respect to $K \cap I=0$, and let $E, \bar{I}, \bar{R}$ denote the images of $E, I, R$ respectively in $R / K$. Then $\bar{E}$ is bi-essential in $\bar{R}$ as $R-R$ subbimodule, hence as $\bar{R}$ ideal, so $\bar{R}$ is bi-artinian from Theorem 7.7. Hence $\bar{I} \simeq I$ is a bi-artinian $R-R$ bimodule, and the result follows. The çase of $A(R)$ is similar.

(ix). This follows by an easy adaption of the proof of (iv).

\section{The Brown-McCoy Radical and Normalising elements}

In this section we temporarily drop our concentration on rings with unity, to look at ringlets and bimodulets over them. The first result shows that this apparent relaxation of conditions is, in a certain sense, not really a relaxation at all.

Proposition 8.1. Let $S, R$ be ringlets and $B$ be an $S-R$ bimodulet such that $B=S u=u R$ for some $u \in B$. Suppose $B$ is right faithful. Then $R$ is a ring, and $B$ is right unital.

Proof: For some $x \in R, u=u x$. If $r \in R, u r=u x r$, and $R A(u)=R A(B)$, so $r=x r$. Also, $u r=s u$ for some $s \in S$, so $u r x=s u x=s u=u r$, and hence $r=r x$. Thus $R$ is a ring, and it is implicit in this argument that $B$ is also right unital.

It is known [26] that the analogue of this result, in which it is only assumed that $B=S a=b R$ for some $a, b \in B$ is false, even if $S=B=R$ is a simple (idempotent) ringlet.

Recall that the term supersimple was defined for bimodulets over ringlets. We say that an $S-R$ bimodulet $B$ is $C$-supersimple if $S=R$ and $B$ is generated by a centralising element. The previous proposition now yields the following annihilator characterisation of the Brown-McCoy radical, parallelling the familiar annihilator characterisation of the Jacobson radical.

THEOREM 8.2. Let $R$ be a ringlet, and $S$ be a 'variable' ringlet. The Brown$M c C o y$ radical $G(R)$ is equal to each of the following four sets:

(i) the set of elements which right annihilate all C-supersimple $R-R$ bimodulets;

(ii) the set of elements which right annihilate all supersimple $S-R$ bimodulets;

(iii) the set of elements which left annihilate all C-supersimple $R-R$ bimodulets;

(iv) the set of elements which left annihilate all supersimple $R-S$ bimodulets. 
Proof: If $B$ is a supersimple right faithful $S-R$ bimodulet, then Proposition 8.1 shows that $R$ is a simple ring with unity. Conversely, if $M$ is an ideal of $R$ such that $R / M$ is a simple ring with unity then $R / M$ is a C-supersimple $R-R$ bimodulet. The result now follows from the standard characterisation of the Brown-McCoy radical [9, Chapter 5].

\section{The INTERSECTION THEOREM}

The previous section again highlights the similarity between the Brown-McCoy and the Jacobson radicals which (for rings) was noted in Section 4. It is therefore of interest to investigate the obvious analogue, for the Brown-McCoy radical, of the Jacobson Conjecture concerning the Jacobson radical of a left-right noetherian ring. Indeed the analogous conjecture is true:

Theorem 9.1. Let $R$ be a BPN ring, with Brown-McCoy radical $G$ and Jacobson radical $J$. Then $\bigcap_{n=1}^{\infty} G^{n}=\bigcap_{n=1}^{\infty} J^{n}=0$.

PROOF: Since $J(R) \subset G(R)$ for any ringlet $R$, it suffices to prove the first claim. It is well-known that it suffices to consider the case in which $R$ is subdirectly irreducible, and that then $G$ must be nilpotent. Now if $R$ is subdirectly irreducible then it is biartinian, by Theorem $7.7(i)$, so has a bimodule composition series with supersimple factors. If $F$ is the intersection of the distinct right annihilators of the supersimple factors, then $F$ is a finite intersection of maximal ideals, and $F^{n}$ right annihilates $R$ for some $n \in \mathrm{N}$. Hence $F$ is nilpotent, so $F=G$ and $G$ is nilpotent, as required.

Another application of the artinian radical of a left-right noetherian ring concerns the direct sum decomposition of a noetherian serial ring. The analogous problem for $\mathrm{BPN}$ rings is the structure of rings in which the ideals are linearly ordered. It is easy to adapt the proof of $[4$, Theorem 2.2$]$ to establish:

Proposition 9.2. Let $R$ be a BPN ring in which the (two-sided) ideals are linearly ordered. Then $R$ is pre-simple, and is either prime or bi-artinian (or both).

Remark. In the bi-artinian case, it is possible to adapt Example 3.5 .5 of [24] to obtain a pre-simple BPC ring with linearly ordered ideals which is not QCPIR. It would be of interest to know if there exists a prime ring with those properties which is not QCPIR. The ring $D_{n}$ mentioned in Section 3 Paragraph 7 is a likely candidate, but we have not been able to determine if it fits the bill.

\section{COMPARISONS OF CLASSES OF RINGS}

Much of our work, both here and elsewhere, has been concerned with similarities between the classes of BPC and of BPN rings and the classes of commutative noetherian 
and of left-right noetherian rings. For example, the existence of an artinian radical, the existence [26] of a reduced rank function, the fact [26] that a prime ideal minimal over an invertible ideal has height at most 1 , the characterisation of certain (bi)module categories as abelian, 'good' behaviour under standard ring extensions (cited in Section 3 from [27]); all of these are sinular features of these several classes of rings (and their (bi)modules). It seems plausible that there is some deeper category-theoretic reason for such similarities, but we have so far failed to find one.

Nevertheless, it is worth citing one or two further results which yield more insight into these similarities. Although some of the features of left-right noetherian rings which were just mentioned depend only on the a.c.c on one side, others-such as the existence of the artinian radical-depend on it being satisfied on both sides. It is reasonable to conjecture that the existence of such extensive similarities is connected to two key features of the defining conditions:

(a) each of the classes mentioned is defined by a 'symmetry condition' in the sense of [24], that is, a condition which

(i) is satisfied by a ring $R$ if and only if it is satisfied by the opposite ring;

(ii) is satisfied by every epimorphic image of a ring $R$ which itself satisfies the condition;

(b) the symmetry condition defining each of the classes is, in some sense, 'finite' on both sides, at least to the extent that two-sided ideals must be left and right finitely-generated in every class mentioned.

The properties of being a QCPIR or an SQCPIR are not only symmetry conditions in the sense of (a), but they too have the same additional quality (b) of 'left-right finiteness'.

Also it is well-known that a ring is right (left) artinian if and only if it has a right (left) composition series. Thus the following theorem and its corollary further highlight the similarity of the various 'symmetry plus finiteness' conditions just listed.

TheOREM 10.1. Let $R$ be a ring.

(a) Suppose $\mathbf{M}=R-\operatorname{Mod}-R$ is the category of $R-R$ bimodules; then:

(i) $R$ is an SQCPIR if and only if there exists $M \in \mathbf{M}$ such that $M$ is right (equivalently, left) faithful and each subbimodule of $M$ is generated by an $R-R$ centralising element;

(ii) $R$ is a $B P C$.ring if and only if there exists $M \in \mathbf{M}$ such that $M$ is right (equivalently, left) faithful and $B P C$;

(iii) the following conditions on $R$ are equivalent:

( $\alpha$ ) $R$ is left-right artinian; 
( $\beta$ ) $R$ has a left and a right composition series;

$(\gamma)$ there exists a left-right faithful bimodule $M \in \mathbf{M}$ which has a left and a right composition series;

(iv) the following conditions on $R$ are equivalent:

( $\alpha$ ) $R$ is left-right noetherian;

( $\beta$ ) there exists a left-right faithful bimodule $M \in \mathbf{M}$ which is leftright noetherian;

(b) suppose that, for any ring $S, \mathbf{B}=\mathbf{B}(S)=S-$ Mod $-R$ is the category of $S-R$ bimodules; then:

(i) $R$ is a $Q C P I R$ if and only if for some ring $S$ there exists $B \in \mathbf{B}$ such that $B$ is right faithful and each subbimodule of $B$ is generated by an $S-R$ normalising element;

(ii) $R$ is a $B P N$ ring if and only if for some ring $S$ there exists $B \in \mathbf{B}$ such that $B$ is right faithful and $B$ is a $B P N$ bimodule.

Proof: Well-known, or straightforward using the results of Section 4.

Corollary 10.2. Let $R$ be a semiprime ring. Then $R$ is (i) left-right artinian, (ii) left-right noetherian, (iii) BPC, (iv) BPN, (v) a $Q C P I R$, or (vi) an SQCPIR if and only if $R$ has a bi-essential ideal $I$ which has the corresponding property as bimodule.

Remarks. 1. In Corollary 10.2, the 'corresponding property' for a bimodule to a ring being left-right artinian is, as noted, for the bimodule to have a left and a right composition series. The endomorphism ring $E$ of an infinite dimensional (right) vector space is a prime subdirectly irreducible ring with heart $H$, which is the left socle and is right senisimple. Also $H$ is both left and right essential, but $R$ is neither left nor right artinian.

2. It is probably significant that each of the symmetry conditions mentioned in Theorem $\mathbf{1 0 . 1}$ is 'left-right finite' in the (albeit rather vague) sense mentioned above. There are three obvious symmetry conditions which are not 'finite' in such a fashion, namely the bi-noetherian and the bi-artinian conditions, and the condition of having a bimodule composition series. The following examples show that for none of these is any analogue of Theorem 10.1 true: even if a ring $R$ has a left-right faithful, simple $R-R$ bimodule, it may satisfy either one of the bi-a.c.c and the bi-d.c.c (but not the other), or it may satisfy neither.

Examples. 1. A ring $R$ that is bi-artinian but not bi-noetherian, with a left-right faithful simple $R-R$ bimodule.

Let $D$ be a division ring and $M=M_{D}$ be a right $D$-vector space of sufficiently large infinite dimension that $E=\operatorname{End}\left(M_{D}\right)$ is not a bi-noetherian ring. Then $E$ is a 
prime, subdirectly irreducible ring with heart $K$, which is a simple left-right faithful $E-E$ bimodule, but $E$ is bi-artinian whilst not bi-noetherian.

2. A ring $S$ that is bi-noetherian, but not bi-artinian, with a left-right faithful simple $S-S$ bimodule.

Let $S$ be the prime, subdirectly irreducible left-right noetherian ring which is not bi-artinian exhibited by Stafford in [22], and let $H$ be its heart. Then $H$ is a simple left-right faithful $S-S$ bimodule, but $S$ is bi-noetherian whilst not bi-artinian. By applying a $G$-functor $G_{t}(-)$ to $S$, for $t>1$, a similar example may be constructed which is neither left nor right noetherian.

Alternatively, let $S$ be any of the rings $R_{n}(n \in \mathrm{N})$ defined in Example 5.5 of [23]. Then $S$ is a prime, subdirectly irreducible ring which is bi-noetherian but not bi-artinian. This example too is neither left nor right noetherian.

3. A ring $T$ that is neither bi-artinian nor bi-noetherian, with a left-right faithful simple $T-T$ bimodule.

Let $w^{\prime}$ be an infinite cardinal such that the set Prev $\left(w^{\prime}\right)$ of cardinals strictly less than $w^{\prime}$ does not have a maximal element, and let $w$ be the successor cardinal to $w^{\prime}$. Let $D$ be a division ring of characteristic 0 , and $V$ be a right $D$-vector space of dimension $w$. Let $F$ be the endomorphism ring of $V_{D}$, and $M=\{f \in$ $\left.F \mid \operatorname{dim}_{D}(\operatorname{Im}(f))<w\right\}$ be its unique maximal ideal. Then $F$ is a prime, subdirectly irreducible ring with heart $L \subseteq M$. Let $T$ be the subring of $F$ generated by $M$ and the subring of (left) multiplications by elements of $Z$. Then $L$ is a simple, left-right faithful $T-T$ bimodule, but $T$ is neither bi-noetherian nor bi-artinian.

Remarks. 3. It would be very interesting to know, for each property in Corollary 10.2 , in what other 'natural' classes of rings not restricted to being semiprime the various claims of the Corollary remain true. We recall that, for example, if $R$ is a BPN ring and $A(R)$ or $B(R)$ is bi-essential then $R$ is left-right artinian or bi-artinian respectively.

4. It would also be of interest to determine whether or not there are any 'onesided' analogues of Theorem 10.1 and its corollary. There are obvious examples of non-noetherian right primitive rings which show that no such analogues can be found for (mere?) modules, but it might be that the existence of a left-right faithful bimodule which was 'nice' on one side forced the ring to be 'nice' too. Inspection of the relevant proofs shows, however, that they all depend fundamentally on suitable ideals or subbimodules being finitely-generated on both sides. We therefore conjecture that the (hypothetical) one-sided analogues are false, and specifically:

Conjecture 10.3. There exists a right primitive, non-right noetherian, ring $R$ with a left-right faithful, simple, right simple, non-left noetherian bimodule ${ }_{R} B_{R}$.

We have, however, failed to either construct or locate such an example. 
5. We note that the properties mentioned in Theorem 10.1(a) are all Morita invariant, while it is known [17, p.318] and [23, p.381], or [5, p.24] that the property of being a QCPIR is not, even for left-right noetherian rings. Theorem $10.1(\mathrm{~b})$ covers a second property-being BPN-which depends only on $S-R$ bimodules not on $R-R$ bimodules. It seems likely that this property too is not Morita invariant, and that a counter-example could be found which is left-right noetherian. Thus we make:

CONJECTURE 10.4. There exists a left-right noetherian ring $R$ which is not a $B P N$ ring such that, for some integer $n>1$, the matrix algebra $M_{n}(R)$ is a $Q C P I R$.

We have failed to find such an example.

6. The properties mentioned in Theorem 10.1(a) are also preserved by extension and contraction under non-singular (see [27]) $G$-functors. It seems likely that a pattern similar to that for Morita invariance holds, for those symmetry conditions which only refer to two-sided ideals, where extensions constructed with $G$-functors replace those constructed using finite matrices. In particular, we conjecture that the normalising but not centralising properties are not preserved by contraction:

CONJECTURE 10.5. There exists a left-right noetherian ring $R$ which is not a $B P N$ ring such that, for some cardinal $t>1$, the overring $G_{t}(R)$ is a $Q C P I R$.

Here too we have failed to find a suitable example. But it does seem possible that $G$-functors could be used to create an analogue, for ideals and selected bimodules, of the Morita theorems for one-sided modules.

7. The analogues for the left-right artinian and left-right noetherian conditions of the results in Section 5 about prime ideals in QCPIRs, SQCPIRs, BPC rings and BPN rings are obviously false: a suitable counter-example is any simple ring $S$ which is neither left nor right noetherian.

8. The obvious 'symmetry plus finiteness' condition which we have not discussed is: a ring $R$ has the property that every ideal is both left and right finitelygenerated. Following [24], we say that a bimodule (ring) in which every subbimodule (ideal) is so generated is left-right semi-noetherian. Every BPN ring is left-right seminoetherian. Clearly, this property of being left-right semi-noetherian is Morita invariant. Moreover, if $R$ is a left-right semi-noetherian ring and $S$ is an extension ring of $R$ generated by a finite subnormalising sequence then ${ }_{R} S_{R}$ is a left-right semi-noetherian $R-R$ bimodule, so certainly $S$ is left-right semi-noetherian as a ring. This suggests that the class of such rings may have interesting properties (as also suggested in [24, 26]). We therefore raise our final questions in this area:

Problem 10.6. Is it true that a ring $R$ is left-right semi-noetherian if and only if there is a left-right faithful $R-R$ bimodule which is left-right semi-noetherian? 
Problem 10.7. Is it true that a ring $R$ is left-right semi-noetherian if and only if every prime ideal is left and right finitely-generated?

Using techniques in [26], these questions can be reduced to analogous ones for rings and bimodules in which every ideal (subbimodule) is left and right cyclic. Thus attempted proofs based on the isomorphism theorems and induction on the number of (module or bimodule) generators are apparently unavailable.

\section{REFERENCES}

[1] M.F. Atiyah and I.G. Macdonald, Introduction to commutative algebra (Addison-Wesley, Reading, Mass., 1969).

[2] A.R. Camina and E.A. Whelan, Linear groups and permutations (Pitman, London, 1985).

[3] A.W. Chatters and C.R. Hajarnavis, Rings with chain conditions (Pitman, London, 1980).

[4] A.W. Chatters, C.R. Hajarnavis and N.C. Norton, 'The Artin radical of a Noetherian ring', J. Austral. Math. Soc. 23 (1977), 379-384.

[5] A.W. Chatters and D.A. Jordan, 'Non-commutative unique factorisation rings', J. London Math. Soc. (2) 33 (1986), 22-32.

[6] I.S. Cohen, 'Commutative rings with restricetd minimum condition', Duke Math. J. 17 (1950), 27-42.

[7] M. Cohen and S. Montgomery, 'The normal closure of a semiprime ring', in Ring Theory: proceedings of the 1978 Antwerp conference, F. van Oystaeyen, ed., pp. 43-59 (Marcel Dekker, New York, 1979).

[8] P.M. Cohn, Albegra 2 (Wiley, Chichester, 1979).

[9] N.J. Divinsky, Rings and Radicals (George Allen and Unwin, London, 1965).

[10] A.G. Heinicke and J.C. Robson, 'Normalising extensions: prime ideals and incomparability', $J$. Algebra 72 (1981), 237-268.

[11] A.G. Heinicke and J.C. Robson, 'Normalising extensions: prime ideals and incomparability', University of Leeds, 1983. (preprint).

[12] A.V. Jategaonkar, 'Localization in noetherian rings', in L.M.S. Lecture Notes Series 98 (Cambridge Universtiy Press, Cambridge, 1986).

[13] I. Kaplansky, 'Elementary divisors and modules', Trans. Amer. Math. Soc. 66 (1949), $464-491$.

[14] T.H. Lenagan, 'Artinian ideals in Noetherian rings', Proc. Amer. Math. Soc. 51 (1975), $499-500$.

[15] J.C. McConnell, 'Localisation in enveloping rings', J. London Math. Soc. 43 (1968), 421-428.

[16] K.R. McLean, 'Principal ideal rings and separability', Proc. London. Math. Soc (3) 45 (1982), 300-318.

[17] Y. Nouazé and P. Gabriel, 'Idéaux premiers de l'algèbre enveloppante d'une algèbre de Lie nilpotente', J. Algebra 6 (1967), 77-99.

[18] D.S. Passman, The algebraic structure of groups and rings (Interscience, New York, 1977).

[19] J.C. Robson, 'Idealizers and hereditary noetherian prime rings', J. Algebra 22 (1972), 45-81.

[20] P.F. Smith, 'On non-commutative regular local rings', Glasgow Math. J. 17 (1976), 98-102.

[21] J.T. Stafford, 'On the ideals of a noetherian ring', University of Leeds, (1984). (preprint) .

[22] E.A. Whelan, 'Quasi-commutative principal ideal rings', Quart. J. Math. Oxford (2) 37 (1986), 375-383.

[23] E.A. Whelan, 'Finite subnormalising extensions of rings', J. Algebra 101 (1986), 418-432.

[24] E.A. Whelan, 'Symmetry conditions in ring and module theory (Ph.D. Thesis)', University of East Anglia. 
[25] E.A. Whelan, 'Reduced rank and normalising elements', J. Algebra 113 (1988), 416-429.

[26] E.A. Whelan, 'An infinite construction in ring theory', Glasgow Math. J. 30 (1988), $349-357$.

[27] E.A. Whelan, 'Extensions of rings with normalising elements'. (in preparation) .

[28] E.A. Whelan, 'Normalising elements and radicals II'. (in preparation) .

[29] E.A. Whelan, 'Direct sum decompositions of conformal rings', Proc. Edinburgh Math. Soc.. (submitted).

[30] K.G. Wolfson, 'An ideal-theoretic characterisation of the ring of all linear transformations', $A$ mer. J. Math. 20 (1953), 353-386.

Added in Proof. There is a gap in the above proof of Theorem 5.3, since if $R$ is (left-right) noetherian it is not then obvious that $P / K$ must be supersimple. It is necessary to show that $L A_{R / K}(P / K)$ is a non-minimal prime (and hence contains a regular, because normalising, element); this follows from [28, Proposition 3.11 and Lemma 4.2].

School of Mathematics and Physics,

University of East Anglia,

Norwich, Norfolk NR4 7TJ

England. 